\title{
Dose and Sample Size Determination for Multi-Drug Combination Studies
}

\author{
Ming Tan, Hong-Bin Fang and Guo-Liang Tian \\ Division of Biostatistics \\ University of Maryland Greenebaum Cancer Center \\ MSTF Suite 261, 10 South Pine Street \\ Baltimore, MD 21201, USA \\ Email: mttan@som.umaryland.edu
}

Summary. Preclinical experiment on multi-drug combination has an increasingly important role in (especially cancer) drug development because of the need to reduce development time and costs. Despite of recent progresses in statistical methods for assessing drug interaction, there is a lack of general method for experimental design for such studies. We propose a general method for determining the doses comprising the combinations and the sample sizes to detect departures from additivity especially in the case of more than two drugs in a semi-parametric statistical model. We utilize the uniform scattered points in the experimental domain to determine the doses comprising the combinations and calculate the sample size so that the power to detect departure from additivity is maximized. To our surprise, such an extension to multi-drug is far more difficult than what it appears when there are three or more drugs involved. Using the general methodology, we derive the combinations and sample size specifically for a common class of drugs to derive the experimental design. In addition, we illustrate the method with the SAHA and Ara-C and Etoposide combination studies.

KEY WORDS: Additive action; Dose-effect; Experimental design; F-Test; Semiparametric model; Synergism; Uniform design. 


\section{Introduction}

The study of the joint action of drugs has a long history in pharmacology and biostatistics (see, e.g., Finney, 1971; and reviews by Berenbaum, 1989; Greco et al., 1995). Synergism/super-additivity (antagonism/sub-additivity) commonly refers to a dose-effect greater (lesser) than what would be predictable based on knowledge of single drugs if they are additive. Although several definitions of additivity exist, the statistical (as opposed to mechanistic) Loewe's additivity has been shown to be a generally applicable definition and has thus been the primary accepted definition in the evaluation of drug synergy/antagonism (Berenbaum, 1989; Greco at al., 1995). The Loewe additivity is implied when the doses in a combination contribute to the dose-effect according to individual drug potencies (see more details in Section 2). Throughout this paper, this definition will be used, and compounds, agents and drugs will all be referred to as drugs. Because of the importance of drug combinations in cancer, antiviral and antimicrobial therapies, research on synergy has recently attracted increased attention from statisticians (see, e.g., Tan et al., 2003; Kong and Lee, 2006; Lee and Kong, 2008; Fang et al., 2008). A statistical approach to the design and analysis of drug combinations becomes a natural choice because of large variations in dose effect observed in the administration of precisely the same dose to virtually genetically identical animals (or even different aliquots). What makes the biological experiment unique is that the inter animal (run-to-run) variation is typically much greater than that in a typical industrial (e.g., mechanical or chemical engineering) experiment. In addition, these experiments are costly and sample sizes (e.g., the number of animals) should not be larger than necessary. Statistical experimental design attempts to control and account for the variation in both the design and the analysis stages. The key statistical design issues are to find the doses in the combinations and determine sample sizes (how many combinations and replications at each combination) to detect departures from additivity. Although a PubMed search of "synergy" generated over 40,000 articles up to the year of 2007 on the subject, few of them address experimental design issues 
such as dose-finding and sample size determination in such experiments.

In designing a combination study, the model of the joint action is typically not well specified and the constituent doses in the combinations are to be found instead of being given. One common feature of more classic designs is allocating the doses of one drug only while keeping the doses of another drug fixed under the assumption of constant relative potency (see, e.g., Finney , 1971; Abdelbasit and Plackett, 1982; Tallarida et al., 1997; Laska et al., 1994; Gennings et al., and 2004; Straetemans et al., 2005). Hence, the design is suboptimal. In addition, by ignoring the three dimensional nature of the dose response surface, these methods examine multiple combinations and are prone to giving false synergistic combinations (Dawson et al., 2000) or missing an apparent interaction at a particular combination due to inadequate statistical power. Recognizing these unique features, recently Tan et al., 2003; and Fang et al., 2008) developed a design based on uniform measures (to select the combinations and calculate the sample size (the number of replicates at the selected combinations) to detect departures from additivity under the more realistic setting of nonconstant relative potency. This design is similar in spirit to a space-filling type design but the dose levels have to be determined instead of being given (page 181-191, Cox and Reed, 2000). Unfortunately, the method is limited to two drug combinations. Contrary to what we expect, the extension of the method to multidrug combination turns out to be a challenge as shown later in this article because of the increased complexity in the additive model and in obtaining uniform scattered points in high dimensional dose regions. This may partially explain why design for three drug combination is not available despite of the need for such studies.

In this article, we propose an experimental design for detecting departures from additivity of multiple drugs in preclinical studies in a general statistical setting. The design is derived by the uniform measures that maximizes the power to detect any overall possible departures of a given magnitude from additivity while minimizing the lack of fit of the model for joint action. Thus we refer the design as maximum power design for brevity. This general framework does not require constant relative potency and is flexible enough 
to include the design to test joint action of multiple drugs from multiple different classes of drugs in both in vitro and in vivo experiments. Section 2 formulates the general statistical model for the joint action of drugs and proposes an $F$-statistic to test if additive action presents. The maximum power design to determine doses and sample sizes for testing joint action based on the general statistical model is derived in $§ 3$. In Section 4, we focus on the experimental design the common log-linear dose-response curve and show why it is so difficult to derive the design with combinations of three or more drugs. Section 5 illustrates the proposed design method with a study on three anticancer drugs, where the proposed method identified a synergistic combination that would have been missed and that is currently being tested in a clinical trial. We conclude with a discussion in $\S 6$ and the more theoretic results on the design are included in the Appendix.

\section{Model formulation and statistical inference framework}

Consider a combination of $k$ drugs $A_{1}, \ldots, A_{k}$, comprising doses $X_{i}$ of $A_{i}(i=1, \ldots, k)$. Dose-response relationships for individual drugs are estimated by regression models and are given by

$$
y=f_{i}\left(X_{i}\right), \quad i=1, \ldots, k,
$$

where $y$ is the dose-effect scaled to be a viability (proportion of cells surviving) or a tumor volume (with some transformation) and $f_{i}\left(X_{i}\right)$ is assumed to be an increasing function of $X_{i}$ in the dose range of interest. Then, the potency of $\operatorname{drug} A_{i}$ relative to $\operatorname{drug} A_{1}$ is the ratio of isoeffective doses of $A_{1}$ and $A_{i}, \rho_{i}\left(X_{i}\right)=X_{1} / X_{i}$ where $f_{i}\left(X_{i}\right)=f_{1}\left(X_{1}\right)$, i.e.,

$$
\rho_{i}\left(X_{i}\right)=f_{1}^{-1} f_{i}\left(X_{i}\right) / X_{i}, \quad i=2, \ldots, k
$$

Note the potency $\rho_{i}\left(X_{i}\right)$ may depend on dose $X_{i}$.

For a given dose combination of $A_{1}, \ldots, A_{k}, \boldsymbol{x}=\left(x_{1}, \ldots, x_{k}\right)^{T}$, denote the combination dose-effect (response) by $f_{\text {com }}\left(x_{1}, \ldots, x_{k}\right)$. According to Loewe's definition, the isoboles 
(isoeffect equation) of the $k$ drugs is defined as (see (1a) in Berenbaum, 1989),

$$
\frac{x_{1}}{X_{1}}+\frac{x_{2}}{X_{2}}+\cdots+\frac{x_{k}}{X_{k}}=\tau
$$

where $X_{i}$ represents the dose of the $i$ th drug alone that yields the same response as the combination $\left(x_{1}, \ldots, x_{k}\right)$, i.e.,

$$
f_{\text {com }}\left(x_{1}, \ldots, x_{k}\right)=f_{1}\left(X_{1}\right)=\cdots=f_{k}\left(X_{k}\right) .
$$

$\tau$ is called the interaction index of the $k$ drugs at the combination of $\boldsymbol{x}=\left(x_{1}, \ldots, x_{k}\right)^{T}$. If $\tau=1$, we say that the $k$ drugs are additive at $\boldsymbol{x}$. The $k$ drugs $A_{1}, \ldots, A_{k}$ are synergistic (antagonistic) at $\boldsymbol{x}$ when $\tau<1(>1)$.

From (2.3) and (2.4), we have that

$$
\begin{aligned}
f_{\text {com }}\left(x_{1}, \ldots, x_{k}\right) & =f_{1}\left(X_{1}\right) \\
& =f_{1}\left(\tau X_{1}\right)+\left[f_{1}\left(X_{1}\right)-f_{1}\left(\tau X_{1}\right)\right] \\
& =f_{1}\left(x_{1}+\frac{X_{1}}{X_{2}} x_{2}+\cdots+\frac{X_{1}}{X_{k}} x_{k}\right)+\left[f_{1}\left(X_{1}\right)-f_{1}\left(\tau X_{1}\right)\right],
\end{aligned}
$$

and the term $\left[f_{1}\left(X_{1}\right)-f_{1}\left(\tau X_{1}\right)\right]=0$ if the joint action of $A_{1}, \ldots, A_{k}$ is additive. Then, the regression line for the combination with additive action of $k$ drugs is

$$
\begin{aligned}
y & =f_{1}\left(x_{1}+\frac{X_{1}}{X_{2}} x_{2}+\cdots+\frac{X_{1}}{X_{k}} x_{k}\right) \\
& =f_{1}\left(x_{1}+\rho_{2}\left(X_{2}\right) x_{2}+\cdots+\rho_{k}\left(X_{k}\right) x_{k}\right),
\end{aligned}
$$

and $\rho_{i}\left(X_{i}\right)$ is a function of $\left(x_{1}, \ldots, x_{k}\right)$ determined by $(2.2)$-(2.4). If the potency $\rho_{i}$ in (2.2) is not a constant, the additive model (2.5) has no closed forms.

Since we generally know little about the joint effect of the combinations before experiments, we consider a general semiparametric model for the joint effect of the $k$ drugs in the experimental domain $\mathcal{S}_{0}$,

$$
y=f_{1}\left(x_{1}+\frac{X_{1}}{X_{2}} x_{2}+\cdots+\frac{X_{1}}{X_{k}} x_{k}\right)+f\left(x_{1}, \ldots, x_{k}\right)+\varepsilon
$$


where the function $f$ is unspecified, $\varepsilon$ is the error term due to variation in experiments and is assumed to be normally distributed with mean 0 and variance $\sigma^{2}$. Then, testing the additive action of the $k$ compounds is equivalent to testing the hypothesis $H_{0}: f=0$.

To derive the experimental design, we first assume that model (2.5) has (or is approximated by) a generalized additive structure, namely, additive after transformation. For example, we have an approximate additive structure with polynomial approximation since the additive model with a non-constant potency has no closed form. As further detailed in Section 4, such approximation can be achieved once the individual dose-response or the class of drugs is given. Suppose that there is a one-to-one invertible transformation: $\left(x_{1}, \ldots, x_{k}\right) \in \mathcal{S}_{0} \longmapsto\left(z_{1}, \ldots, z_{k}\right) \in \mathcal{S}$, by

$$
z_{i}=\varphi_{i}\left(x_{1}, \ldots, x_{k}\right), \quad i=1, \ldots, k
$$

such that the additive model (2.5) can be expressed as

$$
f_{1}\left(x_{1}+\frac{X_{1}}{X_{2}} x_{2}+\cdots+\frac{X_{1}}{X_{k}} x_{k}\right) \approx \alpha_{1} g_{1}\left(z_{1}\right)+\cdots+\alpha_{k} g_{k}\left(z_{k}\right),
$$

where the functions $g_{1}, \ldots, g_{k}$ are linearly independent, and

$$
\max \left|f_{1}\left(x_{1}+\frac{X_{1}}{X_{2}} x_{2}+\cdots+\frac{X_{1}}{X_{k}} x_{k}\right)-\left[\alpha_{1} g_{1}\left(z_{1}\right)+\cdots+\alpha_{k} g_{k}\left(z_{k}\right)\right]\right|<\frac{1}{2} \eta / \mathcal{V}(\mathcal{S}),
$$

where $\eta$ is a given magnitude of synergism or antagonism worth detecting and $\mathcal{V}(\mathcal{S})$ is the volume (or area) of the domain of experiment $\mathcal{S}$ (see below). Then model (2.6) becomes

$$
y \approx \alpha_{1} g_{1}\left(z_{1}\right)+\cdots+\alpha_{k} g_{k}\left(z_{k}\right)+g\left(z_{1}, \ldots, z_{k}\right)+\varepsilon, \quad \text { for }\left(z_{1}, \ldots, z_{k}\right) \in \mathcal{S},
$$

where $g\left(z_{1}, \ldots, z_{k}\right)=f\left(x_{1}, \ldots, x_{k}\right)$, and $\alpha_{1}, \ldots, \alpha_{k}$ are known parameters. To ensure the identifiability of $\alpha_{1}, \ldots, \alpha_{k}$, we further assume that the unspecified function $g\left(z_{1}, \ldots, z_{k}\right)$ satisfies the following orthogonality condition:

$$
\int_{\mathcal{S}} G\left(z_{1}, \ldots, z_{k}\right) g\left(z_{1}, \ldots, z_{k}\right) d z_{1} \cdots d z_{k}=\mathbf{0}
$$

where $G\left(z_{1}, \ldots, z_{k}\right)=\left(g_{1}\left(z_{1}\right), \ldots, g_{k}\left(z_{k}\right)\right)^{T}$. 
Consequently, the experimental design for the $k$ drug combinations is transformed into $\boldsymbol{z}=\left(z_{1}, \ldots, z_{k}\right)^{T}$ in domain $\mathcal{S}$ with model $(2.9)$. When there are $m$ experimental points $\boldsymbol{z}^{(i)}=\left(z_{1}^{(i)}, \ldots, z_{k}^{(i)}\right)^{T}, i=1,2, \ldots, m$, in the experimental domain $\mathcal{S}$, we can obtain the dose-levels $\boldsymbol{x}^{(i)}=\left(x_{1}^{(i)}, \ldots, x_{k}^{(i)}\right)^{T} \in \mathcal{S}_{0}, i=1,2, \ldots, m$, by the inverse transformation in (2.7) for the combination experiments of the $k$ drugs. For a given $\boldsymbol{z}$, the additive action in the mixture of the $k$ drugs is implied if $g(\boldsymbol{z})=0$. When $g(\boldsymbol{z})>0(<0)$, the mixture of the $k$ drugs is synergistic (antagonistic).

Hence, testing the additive action of $k$ drugs is equivalent to testing the hypothesis

$$
H_{0}: \quad g=0 \quad \text { versus } \quad H_{1}: g \neq 0 \text {. }
$$

The test is powered at a given magnitude $\eta(>0)$ of departure from additivity worth detecting. Thus, if

$$
\int_{S} g^{2}(\boldsymbol{z}) d \boldsymbol{z} \leq \eta^{2}
$$

then the combinations of $k$ drugs are still considered additive. The amount $\eta$ is the smallest synergy (antagonism) of significance, which is usually based on medical and pharmacological knowledge and especially investigators' experience in a particular class of drugs.

In fact, the test statistic can be derived based on the common lack of fit test involving least square error estimates under the full model (2.9) and the additive model (2.5). Assume that the $m$ points in the experimental domain are $\boldsymbol{z}^{(1)}, \ldots, \boldsymbol{z}^{(m)}$, and there are $n_{i}$ experiments at the dose-level $\boldsymbol{z}^{(i)}=\left(z_{1}^{(i)}, \ldots, z_{k}^{(i)}\right)^{T} \in \mathcal{S}$ with corresponding responses $y_{i j}, j=1, \ldots, n_{i}, i=1, \ldots, m$. Denote $n=n_{1}+\cdots+n_{m}$. Let $\boldsymbol{y}$ be the $n \times 1$ vector with elements $y_{i j}$ ordered lexicographically and $\mathbf{1}_{k}$ be the $k \times 1$ vector of one. Let $Z$ be the $m \times k$ matrix with $i$ th row $\left(g_{1}\left(z_{1}^{(i)}\right), \ldots, g_{k}\left(z_{k}^{(i)}\right)\right)$, where $g_{i}$ is given by (2.8). Denote $V=U Z\left(Z^{T} U^{T} U Z\right)^{-1} Z^{T} U^{T}, J=U\left(U^{T} U\right)^{-1} U^{T}$ and $U=\operatorname{diag}\left(\mathbf{1}_{n_{1}}, \cdots, \mathbf{1}_{n_{m}}\right)$. Then, if hypothesis $H_{0}$ is true (i.e, the joint action of the $k$ drugs is additive), the statistic for the test of lack of fit

$$
F=\frac{\boldsymbol{y}^{T}(J-V) \boldsymbol{y} /(m-k)}{\boldsymbol{y}^{T}(I-J) \boldsymbol{y} /(n-m)}
$$


has a central $F$-distribution with degrees of freedom $m-k$ and $n-m$ (see Wiens, 1991). When the alternative hypothesis $H_{1}$ holds, the statistic (2.12) has a noncentral $F$-distribution with degrees of freedom $m-k$ and $n-m$ and the noncentrality parameter

$$
\delta=\frac{n}{\sigma^{2}}\left[\int_{\mathcal{S}} g^{2}(\boldsymbol{z}) d \xi(\boldsymbol{z})-\boldsymbol{b}_{g, \xi}^{T} B_{\xi}^{-1} \boldsymbol{b}_{g, \xi}\right] \equiv \frac{n}{\sigma^{2}} \mathcal{B}(g, \xi),
$$

where

$$
B_{\xi}=\int_{\mathcal{S}} G(\boldsymbol{z}) G^{T}(\boldsymbol{z}) d \xi(\boldsymbol{z}), \quad \boldsymbol{b}_{g, \xi}=\int_{\mathcal{S}} G(\boldsymbol{z}) g(\boldsymbol{z}) d \xi(\boldsymbol{z}),
$$

and $\xi$ is the design measure, which is a probability distribution function with mass $p_{i}=$ $n_{i} / n$ at $\boldsymbol{z}^{(i)}, i=1, \ldots, m$.

\section{Experimental design based on uniform measures}

Recall our goal is to select the $m$ combinations of drugs, $\boldsymbol{z}^{(1)}, \ldots, \boldsymbol{z}^{(m)}$ in domain $\mathcal{S}$ such that the power of the $F$-test in (2.12) is maximized at a given type I error level and the variability in modeling joint action is minimized. The power of the test is an increasing function of the noncentrality parameter $\delta$ given by (2.13). The quantity $\mathcal{B}(g, \xi)$ is a function of both $g$ and the design measure $\xi$. Let $\mathcal{F}_{\eta}^{-}$be the class of functions whose element $g$ satisfying conditions (2.10) and (2.11), and $\mathcal{F}_{\eta}^{+}$be the class of functions defined again by $(2.10)$ and $(2.11)$ as in $\mathcal{F}_{\eta}^{-}$but with the inequality in (2.11) reversed. Wiens (1991) proved that for any design $\xi$ there are an $g_{+} \in \mathcal{F}_{\eta}^{+}$and an $g_{-} \in \mathcal{F}_{\eta}^{-}$such that

$$
\mathcal{B}\left(g_{+}, \xi\right) \leq \eta^{2}=\min _{g \in \mathcal{F}_{\eta}^{+}} \mathcal{B}(g, \lambda) \text { and } \mathcal{B}\left(g_{-}, \xi\right) \geq \eta^{2}=\max _{g \in \mathcal{F}_{\eta}^{-}} \mathcal{B}(g, \lambda)
$$

where $\lambda$ is the uniform distribution on $\mathcal{S}$. Since the least squares estimate of $\sigma^{2}$ is $\boldsymbol{y}^{T}(I-V) \boldsymbol{y} /(n-k)$ and

$$
E\left[\frac{\boldsymbol{y}^{T}(I-V) \boldsymbol{y}}{n-k}\right]=\sigma^{2}+\frac{n}{n-k} \mathcal{B}(g, \xi),
$$

we have

Optimality. The uniform design measure $\xi$ maximizes the minimum power of the F-test and minimizes the maximum bias in the estimation of $\sigma^{2}$. 
Therefore, if we choose the design measure $\xi$ to be uniform on $\mathcal{S}$, then the noncentrality parameter $\delta$ is maximized and (2.13) becomes

$$
\delta=\frac{n}{\sigma^{2}} \int_{\mathcal{S}} g^{2}(\boldsymbol{z}) d \boldsymbol{z}
$$

It is worthwhile to point out that the experimental points obtained are the uniformly scattered points by the number theory method (NTM), but not random numbers generated from a uniform distribution. Although they are more difficult to obtain, the uniformly scattered points by NTM are more uniform (with smaller discrepancy) and more efficient than those by random numbers from a uniform distribution (see Appendix for more details). The design where experimental points are uniformly scattered based on a uniform design measure in the experimental domain is called the uniform design proposed by Fang and Wang (see, Fang and Wang, 1994; Fang et al., 2000).

\subsection{Uniform design and uniform scattered points}

To obtain uniform scattered points in the experimental domain, i.e., allocating the combinations for the experiment, potentially any NTM generation may apply. We utilize the U-type design of an $m \times k$ matrix $\mathbf{U}_{m, k}=\left(u_{i j}\right)$ with each column being a permutation of $\{1,2, \ldots, m\}$ (Fang and Wang, 1994). Then the $m$ points on the $k$-dimensional unit cube $\mathbb{C}^{k}=(0,1)^{k}, \mathbf{V}_{m, k}=\left(v_{i j}\right)$, are defined by

$$
v_{i j}=\left(u_{i j}-0.5\right) / m, \quad i=1, \ldots, m ; j=1, \ldots, k .
$$

Let $\mathcal{U}_{m, k}$ denote the set of all $\mathbf{U}_{m, k}$ and $\mathcal{V}_{m, k}$ be the set of all $\mathbf{V}_{m, k}$. Then the uniform design is to choose the $m$ points so that the discrepancy of $V_{m, k}$ is the smallest over all of possible $V_{m, k}$ in $\mathcal{V}_{m, k}$. Many U-type design matrices with smallest discrepancy can be found in Fang (1994).

Based on the uniform scattered points on $\mathbb{C}^{k}$, it is easy to obtain the uniform design on the domain $\mathcal{S}$ and the combinations of the drugs for experiment. Given the single-drug dose response models, the domain $\mathcal{S}$ we are interested is, e.g., from ED20 to ED80, or 
some ranges based on the amount of systemic exposure or growth inhibition of cells in a particular experiment. Suppose that the domain $\mathcal{S}=\left\{\boldsymbol{z}=\left(z_{1}, \ldots, z_{k}\right)^{T}: \quad Z_{j L}<z_{j}<\right.$ $\left.Z_{j H}, j=1, \ldots, k\right\}$. Let $\boldsymbol{v}^{(i)}=\left(v_{1}^{(i)}, \ldots, v_{k}^{(i)}\right)^{T}, i=1, \ldots, m$, are $m$ uniform design points on $\mathbb{C}^{k}$ obtained by the U-type method. The $m$ uniform design points on $\mathcal{S}$ are given by $\boldsymbol{z}^{(i)}=\left(z_{1}^{(i)}, \ldots, z_{k}^{(i)}\right)^{T}$, where

$$
z_{j}^{(i)}=v_{j}^{(i)}\left(Z_{j H}-Z_{j L}\right)+Z_{j L}, \quad j=1, \ldots, k, i=1,2, \ldots, m
$$

Then, the $m$ combinations of $k$ drugs $A_{1}, \ldots, A_{k}$ for the combination experiment can be obtained by the inverse transform of (2.7).

\subsection{Sample size determination}

Suppose that the experimental points are uniformly scattered on the domain $\mathcal{S}$ and the number of runs (experiment units) at each point is the same. The sample sizes (number of experimental units) to detect a given meaningful synergism or antagonism can be calculated at a given significance level $(\alpha)$ and a given power level $(1-\beta)$ based on the $F$-statistic in (2.12). At a given alternative $\eta$, the smallest meaningful difference to be detected, the statistic $F$ has a noncentral $F$-distribution with degrees of freedom $m-k$ and $n-m$ and the noncentral parameter $\delta$ in (3.1). Then the sample size calculation can be raedily implemented with SAS or Splus.

Table 1 provides several illustrative designs for which numbers of experimental units and replications (1-6) with the several different effects to detect at given $\alpha=0.05$ and $1-\beta=80 \%$ for $k=2$ and 3 . From this table, the more replications are at each combination, the less is the total number of experiments. However, there should be at least $k+1$ experimental units for the combination experiments of $k$ drugs and at least one replication at each unit. It is worth noting that with the same replication at each mixture, the total experimental units for detecting departures from additivity in three drug combinations are slightly less than those in two drugs combinations. In practice, we should choose more experimental combinations with less replications for the combination 
study of more drugs. The balance of the number of combinations and the number of replications is based on knowledge about the specific drugs. For example, if the variation among animals is known to be substantial, the number of replications may need to be slightly higher.

\section{[insert Table 1 here]}

In summary, to derive the uniform design for the joint action and analyze the data produced, we (1) obtain dose-response regression models of single drugs from experiments of single drugs. (2) choose the meaningful difference $\eta^{2}$ to be detected and then calculate the number of mixtures $m$ by the method in Section 3.2. The variance $\left(\sigma^{2}\right)$ can be estimated by the pooled measurement variations from the single drug experiments. (3) find $m$ combinations of drugs $A_{1}, \ldots, A_{k}$ based on the maximum power design proposed in Section 3.1. (4) upon completion of the combination experiment, use the $F$ statistic in (2.12) to test the hypothesis of the additive action of the $k$ drugs and statistical models (e.g., B-spline) to fit the dose response surface and the interaction index surface (see Fang et al., 2008).

\section{Designs for a common class of single drug dose-response curves}

Note critical to the unform design method is to be able to derive an approximation of the additive model in (2.8) and to obtain uniformed scattered points in the experimental domain. Such derivation depends on the dose response curve. Different classes of drugs may have different dose-response curves. We focus on the log-linear dose-response curve because it represents a wide class of drugs including antimetabolites, antibiotics, interferons, growth factors, neuropeptide $\mathrm{Y}$, phorbol esters, narcotics and neuronal agonists, hepatotoxins, and cromoglycate. Furthermore, other shapes of dose-responses can be reduced into log-linear curves with some transformations, such as Hill models in studies of drugs causing muscle contraction, neuronal activators, inhibitors of cell proliferation and tumor promoters, and in general toxicology (see Berenbaum, 1989). We show how to 
use the general framework to derive experimental designs for log-linear individual doseresponse curves. Assume that the single dose-response curves of drugs $A, B$ and $C$ are

$$
\begin{aligned}
& y\left(X_{A}\right)=\alpha_{A}+\beta_{A} \log X_{A}, \\
& y\left(X_{B}\right)=\alpha_{B}+\beta_{B} \log X_{B}, \\
& y\left(X_{C}\right)=\alpha_{C}+\beta_{C} \log X_{C},
\end{aligned}
$$

respectively, where $y$ is the dose-effect scaled into viability (proportion of cells surviving) or a tumor volume (with some transformation). Without loss of generality, we assume that $\beta_{C} \leq \beta_{B} \leq \beta_{A}$. The potency $\rho\left(X_{B}\right)$ of $B$ relative to $A$ is

$$
\rho\left(X_{B}\right)=\rho_{0} X_{B}^{\beta_{B} / \beta_{A}-1}, \quad \rho_{0}=\exp \left[\left(\alpha_{B}-\alpha_{A}\right) / \beta_{A}\right]
$$

and the potency $\rho\left(X_{C}\right)$ of $C$ relative to $A$ is

$$
\rho\left(X_{C}\right)=\rho_{1} X_{C}^{\beta_{C} / \beta_{A}-1}, \quad \rho_{1}=\exp \left[\left(\alpha_{C}-\alpha_{A}\right) / \beta_{A}\right]
$$

When $\beta_{C}=\beta_{A}$, the potencies $\rho\left(X_{B}\right)$ and $\rho\left(X_{C}\right)$ above are constant and equal to $\rho_{0}$ and $\rho_{1}$, respectively. In this case, the additive model at combination dose $\left(x_{A}, x_{B}, x_{C}\right)$ is

$$
\begin{aligned}
y\left(x_{A}, x_{B}, x_{C}\right)= & \alpha_{A}+\beta_{A} \log \left(x_{A}+\rho_{0} x_{B}+\rho_{1} x_{C}\right) \\
= & \alpha_{A}+\beta_{A} \log \left(z_{1}\right)+\beta_{A} \log \left[\left(1-\rho_{0}\right) z_{2}+\rho_{0}\right] \\
& +\beta_{A} \log \left[\left(1-\frac{\rho_{1}}{\rho_{0}}\right)\left(1-z_{3}\right)+\frac{\rho_{1}}{\rho_{0}}\right],
\end{aligned}
$$

where

$$
\left\{\begin{aligned}
z_{1} & =x_{A}+x_{B}+x_{C} \\
z_{2} & =\frac{x_{A}}{x_{A}+x_{B}+\rho_{1} x_{C} / \rho_{0}} \\
z_{3} & =\frac{x_{C}}{x_{A}+x_{B}+x_{C}}
\end{aligned}\right.
$$

According to Section 2, the $m$ experimental points $\left\{\left(z_{1}^{(i)}, z_{2}^{(i)}, z_{3}^{(i)}\right), i=1, \ldots, m\right\}$ which maximize the statistical power in detecting synergy should be uniformly scattered in the 
experimental domain $\mathcal{S}=\left\{\left(z_{1}, z_{2}, z_{3}\right): Z_{L}<z_{1}<Z_{H},\left(z_{2}, z_{3}\right) \in \mathbb{V}_{2}\right\}$, where $Z_{L}$ and $Z_{H}$ are the lower and upper limits of the total dose according to drug $A$, respectively, and

$$
\mathbb{V}_{q} \hat{=}\left\{\left(w_{1}, \ldots, w_{q}\right): w_{j}>0, j=1, \ldots, q, \Sigma_{j=1}^{q} w_{j}<1\right\}
$$

The $m$ combinations $\left\{\left(x_{A}^{(i)}, x_{B}^{(i)}, x_{C}^{(i)}\right), i=1, \ldots, m\right\}$ can be obtained by the inverse transformation of (4.7).

When $\beta_{C}<\beta_{A}$, the potency $\rho\left(X_{C}\right)$ depends on the dose-level $X_{C}$. Then, based on (2.5), the additive model at combination dose $\left(x_{A}, x_{B}, x_{C}\right)$ is

$$
y\left(x_{A}, x_{B}, x_{C}\right)=\alpha_{A}+\beta_{A} \log \left[x_{A}+\rho_{0}^{\beta_{A} / \beta_{B}} \rho_{1}^{1-\beta_{A} / \beta_{B}} \psi^{\frac{\beta_{C}\left(\beta_{B}-\beta_{A}\right)}{\beta_{B}\left(\beta_{C}-\beta_{A}\right)}} x_{B}+\rho_{1} \psi x_{C}\right]
$$

where $\psi$ is a function of $\left(x_{A}, x_{B}, x_{C}\right)$ and can be obtained by solving the following equation

$$
\psi=\left[\frac{x_{A}}{\rho_{1}}+\left(\frac{\rho_{0}}{\rho_{1}}\right)^{\beta_{A} / \beta_{B}} \psi^{\frac{\beta_{C}\left(\beta_{B}-\beta_{A}\right)}{\beta_{B}\left(\beta_{C}-\beta_{A}\right)}} x_{B}+\psi x_{C}\right]^{1-\beta_{A} / \beta_{C}} .
$$

As alluded to earlier, the difficult is to find an approximation of the additive model (4.9). Since the right-hand side of (4.10) approximates to

$$
\begin{aligned}
& {\left[\frac{x_{A}}{\rho_{1}}+\left(\frac{\rho_{0}}{\rho_{1}}\right)^{\beta_{A} / \beta_{B}} x_{B}+\left(\frac{\rho_{0}}{\rho_{1}}\right)^{\beta_{A} / \beta_{B}} \frac{\beta_{C}\left(\beta_{B}-\beta_{A}\right)}{\beta_{B}\left(\beta_{C}-\beta_{A}\right)}(\psi-1) x_{B}+\psi x_{C}\right]^{1-\beta_{A} / \beta_{C}}} \\
& =\left[\frac{x_{A}}{\rho_{1}}+\left(\frac{\rho_{0}}{\rho_{1}}\right)^{\frac{\beta_{A}}{\beta_{B}}} \frac{\beta_{A}\left(\beta_{C}-\beta_{B}\right)}{\beta_{B}\left(\beta_{C}-\beta_{A}\right)} x_{B}+\left\{\left(\frac{\rho_{0}}{\rho_{1}}\right)^{\frac{\beta_{A}}{\beta_{B}}} \frac{\beta_{C}\left(\beta_{B}-\beta_{A}\right)}{\beta_{B}\left(\beta_{C}-\beta_{A}\right)} x_{B}+x_{C}\right\} \psi\right]^{1-\beta_{A} / \beta_{C}} \\
& \approx\left[\frac{x_{A}}{\rho_{1}}+\left(\frac{\rho_{0}}{\rho_{1}}\right)^{\frac{\beta_{A}}{\beta_{B}}} \frac{\beta_{A}\left(\beta_{C}-\beta_{B}\right)}{\beta_{B}\left(\beta_{C}-\beta_{A}\right)} x_{B}\right]^{1-\beta_{A} / \beta_{C}} \\
& +\frac{\beta_{C}-\beta_{A}}{\beta_{C}}\left[\frac{x_{A}}{\rho_{1}}+\left(\frac{\rho_{0}}{\rho_{1}}\right)^{\frac{\beta_{A}}{\beta_{B}}} \frac{\beta_{A}\left(\beta_{C}-\beta_{B}\right)}{\beta_{B}\left(\beta_{C}-\beta_{A}\right)} x_{B}\right]^{-\frac{\beta_{A}}{\beta_{C}}}\left[\left(\frac{\rho_{0}}{\rho_{1}}\right)^{\frac{\beta_{A}}{\beta_{B}}} \frac{\beta_{C}\left(\beta_{B}-\beta_{A}\right)}{\beta_{B}\left(\beta_{C}-\beta_{A}\right)} x_{B}+x_{C}\right] \psi \\
& \\
& -\frac{\beta_{A}\left(\beta_{C}-\beta_{A}\right)}{2 \beta_{C}^{2}}\left[\frac{x_{A}}{\rho_{1}}+\left(\frac{\rho_{0}}{\rho_{1}}\right)^{\frac{\beta_{A}}{\beta_{B}}} \frac{\beta_{A}\left(\beta_{C}-\beta_{B}\right)}{\beta_{B}\left(\beta_{C}-\beta_{A}\right)} x_{B}\right]^{-\frac{\beta_{A}}{\beta_{C}}-1}\left[\left(\frac{\rho_{0}}{\rho_{1}}\right)^{\frac{\beta_{A}}{\beta_{B}}} \frac{\beta_{C}\left(\beta_{B}-\beta_{A}\right)}{\beta_{B}\left(\beta_{C}-\beta_{A}\right)} x_{B}+x_{C}\right] \psi^{2},
\end{aligned}
$$


then, an approximate solution of (4.9) is given by

$$
\begin{aligned}
\psi\left(x_{A}, x_{B}, x_{C}\right) \approx & \frac{\beta_{C}^{2} h_{1}\left(x_{A}, x_{B}\right)}{\beta_{A}\left(\beta_{C}-\beta_{A}\right) h_{2}^{2}\left(x_{B}, x_{C}\right)}\left\{\left(1-\frac{\beta_{A}}{\beta_{C}}\right) h_{2}\left(x_{B}, x_{C}\right)-h_{1}^{\frac{\beta_{A}}{\beta_{C}}}\left(x_{A}, x_{B}\right)+\right. \\
& {\left.\left[\left(\frac{\beta_{C}-\beta_{A}}{\beta_{C}} h_{2}\left(x_{B}, x_{C}\right)-h_{1}^{\frac{\beta_{A}}{\beta_{C}}}\left(x_{A}, x_{B}\right)\right)^{2}+\frac{2 \beta_{A}\left(\beta_{C}-\beta_{A}\right)}{\beta_{C}^{2}} h_{2}^{2}\left(x_{B}, x_{C}\right)\right]^{1 / 2}\right\}, }
\end{aligned}
$$

where

$$
\begin{aligned}
& h_{1}\left(x_{A}, x_{B}\right)=\frac{x_{A}}{\rho_{1}}+\left(\frac{\rho_{0}}{\rho_{1}}\right)^{\frac{\beta_{A}}{\beta_{B}}} \frac{\beta_{A}\left(\beta_{C}-\beta_{B}\right)}{\beta_{B}\left(\beta_{C}-\beta_{A}\right)} x_{B} \\
& h_{2}\left(x_{B}, x_{C}\right)=\left(\frac{\rho_{0}}{\rho_{1}}\right)^{\frac{\beta_{A}}{\beta_{B}}} \frac{\beta_{C}\left(\beta_{B}-\beta_{A}\right)}{\beta_{B}\left(\beta_{C}-\beta_{A}\right)} x_{B}+x_{C} .
\end{aligned}
$$

The additive model (4.9) becomes

$$
y\left(x_{A}, x_{B}, x_{C}\right) \approx g_{1}\left(z_{1}\right)+g_{2}\left(z_{2}\right)+g_{3}\left(z_{3}\right)
$$

where

$$
\begin{aligned}
& g_{1}\left(z_{1}\right)=\alpha_{A}+\beta_{A} \log \left(z_{1}\right) \\
& g_{2}\left(z_{2}\right)=\beta_{A} \log \left[\left(1-\rho_{0}\right) z_{2}+\rho_{0}\right], \\
& g_{3}\left(z_{3}\right)=\beta_{A} \log \left[\left(1-\rho_{1} / \rho_{0}\right)\left(1-z_{3}\right)+\rho_{1} / \rho_{0}\right]
\end{aligned}
$$

and

$$
\left\{\begin{aligned}
z_{1}= & x_{A}+\left(\frac{\rho_{0}}{\rho_{1}}\right)^{\frac{\beta_{A}}{\beta_{B}}-1}\left[\psi\left(x_{A}, x_{B}, x_{C}\right)\right]^{\frac{\beta_{A}\left(\beta_{C}-\beta_{B}\right)}{\beta_{B}\left(\beta_{C}-\beta_{A}\right)}} x_{B}+\psi\left(x_{A}, x_{B}, x_{C}\right) x_{C} \\
z_{2}= & \frac{x_{A}}{} \\
& x_{A}+\left(\frac{\rho_{0}}{\rho_{1}}\right)^{\frac{\beta_{A}}{\beta_{B}}-1}\left[\psi\left(x_{A}, x_{B}, x_{C}\right)\right]^{\frac{\beta_{A}\left(\beta_{C}-\beta_{B}\right)}{\beta_{B}\left(\beta_{C}-\beta_{A}\right)}} x_{B}+\frac{\rho_{1}}{\rho_{0}} \psi\left(x_{A}, x_{B}, x_{C}\right) x_{C} \\
z_{3}= & \frac{\psi\left(x_{A}, x_{B}, x_{C}\right) x_{C}}{z_{1}} .
\end{aligned}\right.
$$

Similarly, the $m$ experimental points $\left\{\left(z_{1}^{(i)}, z_{2}^{(i)}, z_{3}^{(i)}\right), i=1, \ldots, m\right\}$ which maximize the statistical power in detecting synergy should be uniformly scattered in the experimental 
domain $\mathcal{S}=\left\{\left(z_{1}, z_{2}, z_{3}\right): Z_{L}<z_{1}<Z_{H}, \quad\left(z_{2}, z_{3}\right) \in \mathbb{V}_{2}\right\}$, and the $m$ combinations $\left\{\left(x_{A}^{(i)}, x_{B}^{(i)}, x_{C}^{(i)}\right), i=1, \ldots, m\right\}$ can be obtained by the inverse transformation of (4.13).

If we consider the combination experiments of only two drugs $A$ and $B$, the additive model at combination dose $\left(x_{A}, x_{B}\right)$ is

$$
y\left(x_{A}, x_{B}\right)=\alpha_{A}+\beta_{A} \log \left(x_{A}+\phi\left(x_{A}, x_{B}\right) x_{B}\right)
$$

where $\phi\left(x_{A}, x_{B}\right)$ can be obtained by solving the following equation

$$
\phi\left(x_{A}, x_{B}\right)=\rho_{0}\left(\phi^{-1}\left(x_{A}, x_{B}\right) x_{A}+x_{B}\right)^{\left(\beta_{B}-\beta_{A}\right) / \beta_{A}} .
$$

Since

$$
\left(\phi^{-1}\left(x_{A}, x_{B}\right) x_{A}+x_{B}\right)^{\left(\beta_{B}-\beta_{A}\right) / \beta_{A}} \approx x_{B}^{\left(\beta_{B}-\beta_{A}\right) / \beta_{A}}+\frac{\beta_{B}-\beta_{A}}{\beta_{A}} x_{B}^{\left(\beta_{B}-2 \beta_{A}\right) / \beta_{A}} x_{A} \phi^{-1}\left(x_{A}, x_{B}\right),
$$

we have

$$
\phi\left(x_{A}, x_{B}\right) \approx \frac{1}{2}\left\{\rho_{0} x_{B}^{\left(\beta_{B}-\beta_{A}\right) / \beta_{A}}+\left(\rho_{0}^{2} x_{B}^{2\left(\beta_{B}-\beta_{A}\right) / \beta_{A}}+4 \rho_{0} x_{A} x_{B}^{\left(\beta_{B}-2 \beta_{A}\right) / \beta_{A}} \frac{\left(\beta_{B}-\beta_{A}\right)}{\beta_{A}}\right)^{1 / 2}\right\}
$$

and

$$
y\left(x_{A}, x_{B}\right) \approx g_{1}\left(z_{1}\right)+g_{2}\left(z_{2}\right),
$$

where $g_{1}\left(z_{1}\right)=\alpha_{A}+\beta_{A} \log \left(z_{1}\right), g_{2}\left(z_{2}\right)=\beta_{A} \log \left[\left(1-\rho_{0}\right) z_{2}+\rho_{0}\right]$ and

$$
\left\{\begin{array}{l}
z_{1}=x_{A}+\frac{1}{2} x_{B}^{\beta_{B} / \beta_{A}}\left\{1+\left(1+\frac{4\left(\beta_{B}-\beta_{A}\right) x_{A}}{\beta_{A} x_{B}^{\beta_{B} / \beta_{A}} \rho_{0}}\right)^{1 / 2}\right\} \\
z_{2}=\frac{x_{A}}{z_{1}}
\end{array}\right.
$$

According to Section 2 , the $m$ experimental points $\left\{\left(z_{1}^{(i)}, z_{2}^{(i)}\right), i=1, \ldots, m\right\}$ which maximize the statistical power in detecting synergy should be uniformly scattered in the experimental domain $\mathcal{S}=\left\{\left(z_{1}, z_{2}\right): Z_{L}<z_{1}<Z_{H}, 0<z_{2}<1\right\}$, and the $m$ combinations 
$\left\{\left(x_{A}^{(i)}, x_{B}^{(i)}\right), i=1, \ldots, m\right\}$ can be obtained by inverse transformation of (4.17),

$$
\left\{\begin{array}{l}
x_{A}=z_{1} z_{2} \\
x_{B}=\left[\frac{\rho_{0}\left(z_{1}-z_{1} z_{2}\right)^{2}}{z_{1}\left(1-z_{2}\right) \rho_{0}+\left(\beta_{B} / \beta_{A}-1\right) z_{1} z_{2}}\right]^{\beta_{A} / \beta_{B}} .
\end{array}\right.
$$

When $\beta_{A}=\beta_{B}$, the potency of $\operatorname{drug} B$ relative to $\operatorname{drug} A$ is $\rho_{0}$ and the additive model is

$$
y=\alpha_{A}+\beta_{A} \log z+\beta_{A} \log \left[\left(1-\rho_{0}\right) \pi_{A}+\rho_{0}\right]
$$

where $z=x_{A}+x_{B}$ and $\pi_{A}=x_{A} / z$. This reduces to the special case discussed in Tan et al. (2003) where the uniform measures design is obtained such that the total doses and the mixing proportions are uniformly scattered on the two-dimensional experimental domain $\mathcal{S}=\left\{\left(z, \pi_{A}\right): Z_{L}<z<Z_{H}, 0<\pi_{A}<1\right\}$.

\section{Example: Vorinostat Combined with Ara-C and Etoposide against HL-60}

To illustrate the methods of experimental design for combination studies, we consider the experiments of Vorinostat (SAHA) combined with Ara-C and Etoposide against HL-60. SAHA is a potent histone deacetylase inhibitor that induces cell growth arrest, differentiation, and apoptosis in transformed cells in culture and inhibits tumor growth in animal models. Ara-C is one of the most active agents available for treating acute leukemias. Etoposide has been shown to be an effective anti-leukemia agent, particularly when given in combination with other chemotherapeutic agents. Etoposide exerts its effects by interfering with topoisomerase II activity, binding to and stabilizing the covalent linkage between topoisomerase II and DNA, and inhibiting the re-ligation of the resultant DNA double strand breaks. The goal of this experiment is to determine the effects of preadministration of SAHA on the pharmacokinetics of Ara-C and Etoposide against the leukemia cell line HL-60 (Shiozawa et al., 2006). In the experiments for single agents, we have 56 observations with doses ranging from $0.1 \mu \mathrm{M}$ to $6 \mu \mathrm{M}$ for SAHA, 56 observations with doses ranging from $0.003 \mu \mathrm{M}$ to $0.6 \mu \mathrm{M}$ of Ara-C, and 64 observations with doses ranging 
from $0.01 \mu \mathrm{M}$ to $10 \mu \mathrm{M}$ of Etoposide. Then, the single dose-response curves for Ara-C, Etoposide and SAHA are, respectively,

$$
\begin{aligned}
& y\left(X_{A}\right)=4.80-12.76 \log \left(X_{A}\right) \\
& y\left(X_{B}\right)=41.52-13.02 \log \left(X_{B}\right) \\
& y\left(X_{C}\right)=54.55-23.98 \log X_{C},
\end{aligned}
$$

where $\mathrm{y}$ is the $100 \times$ viability, and $X_{A}, X_{B}$ and $X_{C}$ are the doses of Ara-C, Etoposide and SAHA respectively. The potency of Etoposide relative to Ara-C is $\rho_{0}\left(X_{B}\right)=0.0563 X_{B}^{0.0204}$ and the potency of SAHA relative to Ara-C is $\rho_{1}\left(X_{C}\right)=0.0203 X_{C}^{0.8793}$, which are nonconstant and depend on dose. The predicted additive model at $\left(x_{A}, x_{B}, x_{C}\right)$ is

$$
y\left(x_{A}, x_{B}, x_{C}\right)=4.80-12.76 \log \left(x_{A}+0.0551 \psi^{0.0427} x_{B}+0.0203 \psi x_{C}\right),
$$

where $\psi$ is determined by

$$
\psi=\left(49.3483 x_{A}+2.7204 \psi^{0.0427} x_{B}+\psi x_{C}\right)^{0.4679} .
$$

An approximate additive model is given by

$$
\begin{aligned}
y_{\text {appr }}\left(x_{A}, x_{B}, x_{C}\right)= & 4.80-12.76 \log \left(z_{1}\right)-12.76 \log \left((1-0.0563) z_{2}+0.0563\right) \\
& -12.76 \log \left((1-0.3601) z_{3}+0.3601\right)
\end{aligned}
$$

where

$$
\left\{\begin{aligned}
z_{1} & =x_{A}+0.9798 \psi^{0.0427}\left(x_{A}, x_{B}, x_{C}\right) x_{B}+\psi\left(x_{A}, x_{B}, x_{C}\right) x_{C} \\
z_{2} & =\frac{x_{A}}{x_{A}+0.9798 \psi^{0.0427}\left(x_{A}, x_{B}, x_{C}\right) x_{B}+0.3601 \psi\left(x_{A}, x_{B}, x_{C}\right) x_{C}} \\
z_{3} & =\frac{\psi\left(x_{A}, x_{B}, x_{C}\right) x_{C}}{z_{1}},
\end{aligned}\right.
$$


and

$$
\begin{aligned}
\psi\left(x_{A}, x_{B}, x_{C}\right)= & \frac{4.0166 h_{1}\left(x_{A}, x_{B}\right)}{h_{2}^{2}\left(x_{B}, x_{C}\right)}\left\{0.4679 h_{2}\left(x_{B}, x_{C}\right)-h_{1}^{0.5321}\left(x_{A}, x_{B}\right)\right. \\
& \left.+\left[\left(0.4679 h_{2}\left(x_{B}, x_{C}\right)-h_{1}^{0.5321}\left(x_{A}, x_{B}\right)\right)^{2}+0.4979 h_{2}^{2}\left(x_{B}, x_{C}\right)\right]^{1 / 2}\right\}, \\
h_{1}\left(x_{A}, x_{B}\right)= & 49.3483 x_{A}+2.6042 x_{B}, \\
h_{2}\left(x_{B}, x_{C}\right)= & 0.1164 x_{B}+x_{C},
\end{aligned}
$$

and this approximation is very well since $\max \left\{\left|y\left(x_{A}, x_{B}, x_{C}\right)-y_{\text {appr }}\left(x_{A}, x_{B}, x_{C}\right)\right|: 0.0005 \leq\right.$ $\left.x_{A} \leq 0.2, \quad 0.05 \leq x_{B} \leq 3.2, \quad 0.3 \leq x_{C} \leq 3.2\right\} \leq 0.26$.

To obtain uniform design for testing the joint action of Ara-C, Etoposide and SAHA , the dose range is chosen such that the endpoint, $100 \times$ viability, is from 20 to 80 for Ara-C. Then, the total dose ranges from $0.0028 \mu \mathrm{m}$ to $0.3038 \mu \mathrm{m}$ in Ara-C. The pooled variance from the two single drug experiments is 988.422. For a meaningful difference $\eta$ of 15 $(100 \times$ viability) and 5 replications for each mixture, with type I error rate 0.05 and power 0.80, we need study 21 mixtures in the experiment in order to detect synergy/antagonism in the combination of ara-C, Etoposide and SAHA (total 105 experiments). The U-type matrix with 21 experiment units is given by

$\mathbf{U}_{21,3}=\left(\begin{array}{ccccccccccccccccccccc}1 & 2 & 3 & 4 & 5 & 6 & 7 & 8 & 9 & 10 & 11 & 12 & 13 & 14 & 15 & 16 & 17 & 18 & 19 & 20 & 21 \\ 6 & 16 & 12 & 1 & 20 & 9 & 18 & 4 & 11 & 15 & 3 & 21 & 8 & 14 & 10 & 5 & 19 & 2 & 17 & 7 & 13 \\ 9 & 18 & 2 & 14 & 5 & 21 & 11 & 4 & 16 & 7 & 19 & 13 & 1 & 15 & 8 & 12 & 20 & 6 & 3 & 17 & 10\end{array}\right)^{T}$

and its central $L_{2}$-discrepancy is 0.001939 (see (A3) in Appendix). With the algorithm given in Appendix, we get 21 points in domain $\left\{\left(z_{1}, z_{2}, z_{3}\right)^{T}: 0.0028<z_{1}<\right.$ 0.3038, $\left.\left(z_{2}, z_{3}\right) \in \mathbb{V}_{2}\right\}$. According to (5.4), 21 mixtures of these three drugs for experiments are given in Table 2, of which the doses of Etoposide and SAHA are $16.78149\left(x_{B}^{(i)}\right)^{0.98}$ and $7.961724\left(x_{C}^{(i)}\right)^{0.5321}$, respectively, because of the total dose range according to Ara-C. [insert Table 2 here]

\section{Discussion}

Utilizing information on the single dose-response curves, we have proposed a maximum 
power statistical design for testing drug synergism based on a general model for joint action of drugs where no-specific parametric form of the synergistic/antagonistic effect is assumed. An $F$-test is proposed for detecting synergism/antagonism of drugs in the experimental domain. Selecting doses that comprise the combinations uniformly scattered in the experimental domain maximizes the minimum power of the $F$-test for detecting departure from additivity. The power optimality is derived from the properties of uniform measures and by minimizing the variability in modeling the dose-effect while allocating the combinations reasonably to obtain best possible estimate of the dose response surface of the joint action. In fact, the uniform design for generating experimental combinations (the doses of each drug) using the quasi-Monte Carlo methods is an optimal fractional factorial design under a general majorization framework with exponential kernels (Zhang, 2005; Zhang et al., 2005). Hickernell et al. (2005) showed using quasi-Monte Carlo methods instead of the Monte Carlo method usually improves accuracy of computing the integral of a function.

In drug combination studies, the fixed ratio or ray design chooses total doses based on an assumed dose-effect while fixing the ratio of doses of the two compounds. When the individual dose-response curves are linear in $\log$ dose, the proposed design extends the optimal design by Abdelbasit and Plackett (1982) and the uniform design (see Tan et al., 2003). In general, the (especially, non-constant) relative potency of drugs is ignored in the fixed ratio design, which results in a non-uniformly scattered of combinations and the power to detect synergism is undermined. In addition, the fixed ratio design may miss an apparent interaction at a particular combination and is inadequate for the combination studies of three or more drugs.

More importantly, the number of experimental units and replicates (sample size) in the proposed design is feasible for both in vitro and/or in vivo experiments. Table 1 shows that the sample sizes for detecting departures for additivity of three drugs are not greater than those of two drugs. However, if the F-test shows that there is a synergism/antagonism of the three drugs, the regression analysis for fitting the response surface to find the best 
synergistic mixture of drugs may need more experimental combinations because there are more variables in the model. Hence, the precision of the estimated dose-response may need to be factored in determining the number of experimental mixtures and the number of replications at each combination should be considered.

However, despite of the general framework for the design of combination studies, it is critical that we can identify $k$ linear independent functions $g_{1}\left(z_{1}\right), \ldots, g_{k}\left(z_{k}\right)$ that achieve good approximation in (2.8) and obtain uniformed scattered points in the experimental domain. As shown in the SAHA combined with Ara-C and Etoposide example, individual dose-response curves and relative potency should be incorporated in each specific experiment to maximize the efficiency of the design. Finally, more efficient experimental design may be derived by using results from previous experiments on similar compounds, where the assumption of certain specific dose-response relationships may become plausible in light of prior data. Therefore, a more traditional (such as D-) optimal design or a Bayesian design may be utilized in this case.

\section{ACKNOWLEDGMENTS}

This research was supported in part by US National Institutes of Health grant CA106767. The authors thank Douglas D. Ross, MD, Ph.D. for inclusion of the SAHA combination study as an example.

\section{REFERENCES}

Abdelbasit, K. M., Plackett, R. L. (1982). Experimental design for joint action. Biometrics 38, 171-179.

Berenbaum, M. C. (1989). What is synergy? Pharmcological Reviews 41, 93-141.

Chou, T. C. and Talalay, P. (1984). Quantitative analysis of dose-effect relationships: the combined effects of multiple drugs or enzyme inhibitors. Adv. Enzyme Regul. 22, 27-55. 
Copenhaver, T. W., Lin, T.L., and Goldberg, K.M. (1987). Joint drug action: a review. American Statistical Association, Proceedings of the Biopharmaceutical Section, 160164.

Cox, D. R. and Reid, N. (2000). The Theory of the Design of Experiments. Chapman and Hall/CRC, London.

Dawson, K. S., Carter, W. H. Jr., and Gennings, C. (2000). A statistical test for detecting and characterizing/departures from additivity in drug/chemical combinations. J. Agric. Bio. and Enviro. Statistics 5, 342-359.

Fang, H. B., Ross, D. D., Sausville, E. and Tan, M. (2008). Experimental design and interaction analysis of combination studies of drugs with log-linear dose responses. Statistics in Medicine, DOI: 10.1002/sim.3204

Fang, K. T. (1994). Uniform design and Uniform Design Tables, Beijing: Science Press.

Fang, K. T., Lin, D. K. J., Winker, P. and Zhang, Y. (2000). Uniform design: theory and application. Technometrics 42, 237-248.

Fang, K. T. and Wang, Y. (1994). Number-Theoretic Methods in Statistics. Chapman and Hall, London.

Finney, D. J. (1971). Probit Analysis. 3rd edn. Cambridge University Press, London.

Gennings, C. and Carter, W. H. Jr. (1995). Utilizing concentration-response data from individual components to detect statistically significant departures from additivity in chemical mixtures. Biometrics 51, 1264-1277.

Gennings, C., Carter, W. H. Jr., Carney, E. W., Charles, G. D., Gollapudi, B. B., and Carchman, R. A. (2004). A novel flexible approach for evaluating fixed ratio mixtures of full and partial agonists. Toxicol. Sci. 80, 134-150.

Giltinan, D. M., Capizzi, T. P., and Malan, H. (1988). Diagnostic tests for similar action of two compounds. Applied Statistics 37, 39-50.

Greco, W. R., Bravo, G. and Parsons, J. C. (1995). The search for synergy: a critical review from a response surface perspective. Pharmcological Reviews 47, 331-385.

Greco, W. R., Park, H. S., and Rustum, Y. M. (1990). An application of a new approach 
for the quantitation of drug synergism to the combination of cis-diamminedichloroplatinum and 1- $\beta$-D-arabinofuranosylcytosine. Cancer Res. 50, 5318-5327.

Hickernell, F. J. (1998). A generalized discrepancy and quadrature error bound. Mathematics of Computation 67, 299-322.

Hickernell, F. J., Lemieux, C. and Owen, A. B. (2005). Control variates for quasi-Monte Carlo (with discussions). Statistical Science 20, 1-31.

Hlawka, E. (1962). Zur angenäherten Berechnung mehrfacher Integrale. Monatsh. Math. 66, 140-151.

Kiefer, J. (1961). On large deviations of the empiric d.f. of vector chance variables and a law of the iterated logarithm. Pacific J. Math. 11, 649-660.

Kong , M. and Lee, J. J. (2006). A generalized response surface model with varying relative potency for assessing drug interactions. Biometrics, 62, 986-995.

Korobov, N. M. (1959). The approximate computation of multiple integrals. Dolk. Nauk SSSR 124, 1207-1210.

Laska, E. M., Meisner, M. and Siegel, C. (1994). Simple designs and model-free tests for synergy. Biometrics 50, 834-841.

Lee, J. J. and Kong, M. (2008). Confidence Intervals of Interaction Index for Assessing Multiple Drug Interaction, Statistics in Biopharmaceutical Research, in press.

Loewe, S. (1955). Isobols of dose-effect relations in the combination of pentylenetetrazole and phenobarbital. J. Pharmacol. Exp. Ther. 114, 185-191.

Shiozawa, K., Nakanishi, T., Tan, M., Fang, H. B., Edelman, M. J., Gojo, I., Sausville, E., and Ross, D. D. (2006). Schedule Dependent Synergy of Suberoylanilide Hydroxamic Acid (SAHA or Vorinostat) in Combination with Cytarabine (ara-C) and Etoposide in HL-60 Human Acute Myeloid Leukemia Cells. American Association for Cancer Research 97th Annual Meeting, Washington DC.

Straetemans, R., O’Brien, T., Wouters, L., van Dun, J., Janicot, M., Bijnens, L., Burzykowski, T., and Aerts, M. (2005). Design and analysis of drug combination experiments. Biometrical Journal 47, 299-308. 
Tallarida, R. J., Stone, D. J., and Raffa, R. B. (1997). Efficient designs for studying synergistic drug combinations. Life Science 61, 417-425.

Tan, M., Fang, H.B., Tian, G. L. and Houghton, P. J. (2003). Experimental design and sample size determination for testing synergy in drug combination studies based on uniform measures. Statistics in Medicine 22, 2091-2100.

Wiens, D. P. (1991). Designs for approximately linear regression: two optimality properties of uniform designs. Statistics \& Probability Letters 12, 217-221.

Zhang, A. (2005). Schur-Convex discrimination of designs using power and exponential kernels. In Contemporary Multivariate Analysis and Experimental Design (J. Fan and G. Li eds), 293-311, Singapore: World Scientific Publisher.

Zhang, A., Fang, K. T., Li, R. and Sudjianto, A. (2005). Majorization framework for balanced lattice designs. Ann. Statist. 33, 2837-2853.

\section{APPENDIX: Uniform Design}

The uniform design is proposed by Fang and Wang (see, Fang and Wang, 1994; Fang et $a l ., 2000)$ and has been widely used in industrial experiments. The uniform design points are a class of uniformly scattered points in the experimental domain. Suppose that there are $m$ points in the $k$-dimensional unit cube $\mathbb{C}^{k}=(0,1)^{k}$, i.e. $\mathcal{P}_{m}=\left\{\boldsymbol{w}^{(1)}, \ldots, \boldsymbol{w}^{(m)}\right\} \subset$ $\mathbb{C}^{k}$. Let $F_{m}(\boldsymbol{w})$ be the empirical distribution function of $\mathcal{P}_{m}$,

$$
F_{m}(\boldsymbol{w})=\frac{1}{m} \sum_{i=1}^{m} I\left\{\boldsymbol{w}^{(i)} \leq \boldsymbol{w}\right\}
$$

where $I\{\cdot\}$ is the indicator function and all inequalities are componentwise. The discrepancy of $\mathcal{P}_{m}$ can be defined by

$$
D\left(\mathcal{P}_{m}\right)=\sup _{\boldsymbol{w} \in \mathbb{C}^{k}}\left|F_{m}(\boldsymbol{w})-F(\boldsymbol{w})\right|,
$$

where $F(\boldsymbol{w})$ is the uniform distribution function on $\mathbb{C}^{k}$. This discrepancy is the SmirnoKolmogorov statistic for goodness-of-fit tests and has been universally accepted in quasiMonte Carlo methods. However, the discrepancy defined in (A2) is hard to computation. 
In practice, an equivalent form, the central $L_{2}$-discrepancy proposed by Hickernell (1998),

$$
\begin{aligned}
\left(C L_{2}\left(\mathcal{P}_{m}\right)\right)^{2}= & \left(\frac{13}{12}\right)^{k}-\frac{2}{m} \sum_{i=1}^{m} \prod_{j=1}^{k}\left(1+\frac{1}{2}\left|w_{i j}-0.5\right|-\frac{1}{2}\left|w_{i j}-0.5\right|^{2}\right) \\
& +\frac{1}{m} \sum_{i=1}^{m} \sum_{l=1}^{m} \prod_{j=1}^{k}\left[1+\frac{1}{2}\left|w_{i j}-0.5\right|+\frac{1}{2}\left|w_{l j}-0.5\right|-\frac{1}{2}\left|w i j-w_{l j}\right|\right],
\end{aligned}
$$

is used, where $\boldsymbol{w}^{(i)}=\left(w_{i 1}, \ldots, w_{i k}\right)^{T}$ (see, e.g. Fang et al., 2000).

Obviously, the smaller the discrepancy, the more uniformly scattered on $\mathbb{C}^{k}$ the set of points $\mathcal{P}_{m}$ is. Kiefer (1961) proved that if $\boldsymbol{w}^{(1)}, \ldots, \boldsymbol{w}^{(m)}$ are i.i.d according to the uniform distribution on $\mathbb{C}^{k}$, then

$$
\left.D\left(\mathcal{P}_{m}\right)=O\left(m^{-1 / 2}(\log \log m)^{1 / 2}\right)\right),
$$

with probability one. However, Korobov (1959) and Hlawka (1962) pointed out independently that for a given prime number $m$, using the number-theoretic method (the good-lattice-point method), we can choose $m$ points $\mathcal{P}_{m}=\left\{\boldsymbol{w}^{(1)}, \ldots, \boldsymbol{w}^{(m)}\right\} \subset \mathbb{C}^{k}$ such that its discrepancy

$$
D\left(\mathcal{P}_{m}\right)<c(k) m^{-1}(\log m)^{k}
$$

where the constant $c(k)$ depends on $k$ only. This fact indicates that $\mathcal{P}_{m}$ generated by the Monte Carlo method is not uniformly scattered on $\mathbb{C}^{k}$ with probability one.

The aim of the uniform design is to choose a set of $m$ points $\mathcal{P}_{m}$ in $\mathbb{C}^{k}$ with smallest discrepancy value $D\left(\mathcal{P}_{m}\right)$. If we can find a sequence $\mathcal{P}_{m}$ with $D\left(\mathcal{P}_{m}\right)$ having order $O\left(m^{-1}(\log m)^{k-1}\right)$ as $m \rightarrow \infty$, we can consider $\mathcal{P}_{m}$ to be uniformly scattered over $C^{k}$ at least for large $m$. According to the good-lattice-point method, Fang and Wang (1994) proposed a method for generating uniform design points in $C^{k}$ using the U-type matrices and the corresponding discrepancy has order $O\left(m^{-1}(\log m)^{k-1}\right)$ based on numerical comparisons for $k \leq 12$.

Let $\mathbf{U}_{m, k}=\left(u_{i j}\right)$ be an $m \times k$ matrix, where each column is a permutation of $\{1,2, \ldots, m\}$. Its induced matrix, $\mathbf{V}_{m, k}=\left(v_{i j}\right)$, is defined in (3.2). The matrix $V_{m, k}$ 
can be considered as $m$ points on $\mathbb{C}^{k}$. Then the uniform design is to choose the $m$ points so that the discrepancy of $V_{m, k}$ is the smallest over all of possible $V_{m, k}$ and the corresponding $\mathbf{U}_{m, k}$ is called a U-type design matrix. Most of U-type design matrices can be found in Fang (1994) or at http://www.math.hkbu.edu.hk/UniformDesign/.

With uniform design points in $\mathbb{C}^{k}$, we can generate uniformly scattered points in some convex polyhedrons using transformations. The following theorem is used to generate uniformly scattered points in a $k$-dimensional triangular prism $(k \geq 3)$.

Theorem. Let $\mathbb{S}\left(\boldsymbol{a}_{1}, \ldots, \boldsymbol{a}_{6}\right)$ denote a triangular prism in $\mathbb{R}^{k}(k \geq 3)$ with six vertices: $\boldsymbol{a}_{j}=\left(a_{1 j}, \ldots, a_{k j}\right)^{T}, j=1, \ldots, 6$. Assume that the random vector $\boldsymbol{x}=\left(x_{1}, \ldots, x_{k}\right)^{T}$ has the following stochastic representation

$$
\boldsymbol{x}=\left(\boldsymbol{a}_{1}, \ldots, \boldsymbol{a}_{6}\right)\left(\begin{array}{c}
\left(1-y_{1}-y_{2}\right)\left(1-y_{3}\right) \\
y_{1}\left(1-y_{3}\right) \\
y_{2}\left(1-y_{3}\right) \\
\left(1-y_{1}-y_{2}\right) y_{3} \\
y_{1} y_{3} \\
y_{2} y_{3}
\end{array}\right) .
$$

If (i) $\left(y_{1}, y_{2}\right)^{T} \sim g_{12}\left(y_{1}, y_{2}\right),\left(y_{1}, y_{2}\right)^{T} \in \mathbb{V}_{2}$; (ii) $y_{3} \sim g_{3}\left(y_{3}\right), 0<y_{3}<1$; (iii) $\left(y_{1}, y_{2}\right)^{T}$ is independent of $y_{3}$; (iv) the Jacobian $J\left(\boldsymbol{x} \rightarrow y_{1}, y_{2}, y_{3}\right)=\left|H H^{T}\right|^{1 / 2}$ can be decomposed as $c \cdot g_{12}\left(y_{1}, y_{2}\right) g_{3}\left(y_{3}\right)$, where $c$ is a constant and $H=\left(\frac{\partial x_{j}}{\partial y_{i}}\right)_{3 \times k}$; then $\boldsymbol{x}$ is uniformly distibuted in $\mathbb{S}\left(\boldsymbol{a}_{1}, \ldots, \boldsymbol{a}_{6}\right)$.

Specially,

$$
\mathbb{S}\left(\boldsymbol{a}_{1}, \ldots, \boldsymbol{a}_{6}\right)=\left\{\left(x_{1}, x_{2}, x_{3}\right)^{T}:\left(x_{1}, x_{2}\right)^{T} \in \mathbb{V}_{2}, a<x_{3}<b\right\},
$$

with six vertices $\boldsymbol{a}_{1}=(0,0, a)^{T}, \boldsymbol{a}_{2}=(1,0, a)^{T}, \boldsymbol{a}_{3}=(0,1, a)^{T}, \boldsymbol{a}_{4}=(0,0, b)^{T}, \boldsymbol{a}_{5}=$ $(1,0, b)^{T}$, and $\boldsymbol{a}_{6}=(0,1, b)^{T}$. From (A.6), we have $x_{1}=y_{1}, x_{2}=y_{2}$ and $x_{3}=a\left(1-y_{3}\right)+$ $b y_{3}$. Thus, the Jacobian $J\left(\boldsymbol{x} \rightarrow y_{1}, y_{2}, y_{3}\right)=|H|=b-a$. According to above theorem, if $\left(y_{1}, y_{2}\right)^{T} \sim U\left(\mathbb{V}_{2}\right)$ and $y_{3} \sim U(0,1)$, then $\boldsymbol{x} \sim U(\mathbb{S})$. We have the following algorithm to generate $m$ points which are uniformly scattered in the domain $\mathbb{S}$. 


\section{Algorithm:}

1) Generate $m$ points scattered uniformly in $\mathbb{C}^{3}$ using the U-type design (Fang and Wang, 1994). Let $\mathbf{U}_{m, 2}=\left(u_{i j}\right)$ be an $m \times 2$ U-type design matrix, where each column is a permutation of $\{1,2, \ldots, m\}$. Then, $\left\{\left(v_{1}^{(j)}, v_{2}^{(j)}, v_{3}^{(j)}\right)^{T}: v_{i}^{(j)}=\left(u_{i j}-0.5\right) / m, i=\right.$ $1,2,3 ; j=1, \ldots, m\}$ are the $m$ points scattered uniformly in $\mathbb{C}^{3}$.

2) Obtain $m$ points in $\left(y_{1}, y_{2}, y_{3}\right)^{T}$-scale

$$
\left\{\begin{array}{l}
y_{1}^{(j)}=v_{1}^{(j)} \sqrt{v_{2}^{(j)}}, \\
y_{2}^{(j)}=\left(1-v_{1}^{(j)}\right) \sqrt{v_{2}^{(j)}}, \quad j=1, \ldots, m . \\
y_{3}^{(j)}=v_{3}^{(j)}
\end{array}\right.
$$

3) Obtain $m$ points in $\left(x_{1}, x_{2}, x_{3}\right)^{T}$-scale

$$
\left(\begin{array}{c}
x_{1}^{(j)} \\
x_{2}^{(j)} \\
x_{3}^{(j)}
\end{array}\right)=\left(\begin{array}{llllll}
0 & 1 & 0 & 0 & 1 & 0 \\
0 & 0 & 1 & 0 & 0 & 1 \\
a & a & a & b & b & b
\end{array}\right)\left(\begin{array}{c}
\left(1-y_{1}^{(j)}-y_{2}^{(j)}\right)\left(1-y_{3}^{(j)}\right) \\
y_{1}^{(j)}\left(1-y_{3}^{(j)}\right) \\
y_{2}^{(j)}\left(1-y_{3}^{(j)}\right) \\
\left(1-y_{1}^{(j)}-y_{2}^{(j)}\right) y_{3}^{(j)} \\
y_{1}^{(j)} y_{3}^{(j)} \\
y_{2}^{(j)} y_{3}^{(j)}
\end{array}\right), j=1, \ldots, m .
$$




\section{Table 1}

Numbers of mixtures*

\begin{tabular}{|c|c|c|c|c|c|c|}
\hline \multirow{2}{*}{$\begin{array}{l}\text { Meaningful difference } \eta^{2} \\
\qquad\left(d=\eta^{2} / \sigma^{2}\right)\end{array}$} & \multicolumn{6}{|c|}{$\#$ of Replications $n_{0}$} \\
\hline & 1 & 2 & 3 & 4 & 5 & 6 \\
\hline & \multicolumn{6}{|c|}{ for the combination experiments of two drugs } \\
\hline$d=0.3$ & $107(214)$ & $40(120)$ & $21(84)$ & $14(70)$ & $10(60)$ & $7(49)$ \\
\hline$d=0.4$ & $68(136)$ & $25(75)$ & $14(56)$ & $9(45)$ & $3(18)$ & $3(21)$ \\
\hline$d=0.5$ & $48(96)$ & $18(54)$ & $10(40)$ & $6(30)$ & $3(18)$ & $3(21)$ \\
\hline \multirow[t]{2}{*}{$d=0.8$} & $24(48)$ & $9(27)$ & $4(16)$ & $3(15)$ & $3(18)$ & $3(21)$ \\
\hline & \multicolumn{6}{|c|}{ for the combination experiments of three drugs } \\
\hline$d=0.3$ & $105(210)$ & $39(117)$ & $21(84)$ & $13(65)$ & $9(54)$ & $6(42)$ \\
\hline$d=0.4$ & $66(132)$ & $24(72)$ & $13(52)$ & $8(40)$ & $4(24)$ & $4(28)$ \\
\hline$d=0.5$ & $47(94)$ & $17(51)$ & $9(36)$ & $4(20)$ & $4(24)$ & $4(28)$ \\
\hline$d=0.8$ & $23(46)$ & $7(21)$ & $4(16)$ & $4(20)$ & $4(24)$ & $4(28)$ \\
\hline
\end{tabular}




\section{Table 2}

21 mixtures of Ara-C, Etoposide and SAHA for combination experiment

\begin{tabular}{|c|c|c|c|c|c|c|c|}
\hline $\begin{array}{l}\text { Exper. } \\
\#\end{array}$ & $\begin{array}{r}\text { Ara-C } \\
(\mu \mathrm{M})\end{array}$ & $\begin{array}{r}\text { Etoposide } \\
\qquad(\mu \mathrm{M})\end{array}$ & $\begin{array}{r}\text { SAHA } \\
(\mu \mathrm{M})\end{array}$ & $\begin{array}{l}\text { Exper. } \\
\#\end{array}$ & $\begin{array}{r}\text { Ara-C } \\
(\mu \mathrm{M})\end{array}$ & $\begin{array}{r}\text { Etoposide } \\
\qquad(\mu \mathrm{M})\end{array}$ & $\begin{array}{r}\text { SAHA } \\
(\mu \mathrm{M})\end{array}$ \\
\hline 1 & 0.0012 & 0.0933 & 0.4749 & 12 & 0.1248 & 0.6983 & 0.3619 \\
\hline 2 & 0.0059 & 1.2214 & 1.4168 & 13 & 0.0094 & 2.7681 & 0.9663 \\
\hline 3 & 0.1883 & 0.4162 & 1.1650 & 14 & 0.0850 & 0.9432 & 1.7338 \\
\hline 4 & 0.0005 & 0.2020 & 1.4923 & 15 & 0.0450 & 1.7054 & 1.8895 \\
\hline 5 & 0.0283 & 0.6632 & 0.3619 & 16 & 0.0224 & 1.2908 & 2.6776 \\
\hline 6 & 0.0204 & 0.2379 & 1.5646 & 17 & 0.0052 & 0.5223 & 0.4749 \\
\hline 7 & 0.0523 & 0.5928 & 0.7225 & 18 & 0.0065 & 2.2552 & 2.5879 \\
\hline 8 & 0.0138 & 0.0933 & 0.4749 & 19 & 0.0692 & 3.0748 & 0.9663 \\
\hline 9 & 0.0388 & 0.5928 & 1.6679 & 20 & 0.0471 & 1.1172 & 3.0914 \\
\hline 10 & 0.0475 & 1.1867 & 1.0697 & 21 & 0.0981 & 2.0494 & 2.0626 \\
\hline 11 & 0.0081 & 0.3451 & 2.6776 & & & & \\
\hline
\end{tabular}

